\title{
PAL 1: A GLOBULAR CLUSTER ANYWAY?
}

\author{
J. BORISSOVA AND N. SPASSOVA \\ Institute of Astronomy \\ Bulgarian Academy of Sciences, 12 Tzarigradsko shosse, BG- \\ 1784 Sofia, Bulgaria, E-mail: Borisova@bgearn.bitnet
}

CCD photometry of the halo cluster Pal1 in Thuan-Gunn system is presented. The color - magnitude diagrams (Fig. 1) show a well defined red horizontal branch, lower giant branch and main-sequence down to about two magnitudes below the main-sequence turnoff. The giant branch is absent and the brightest stars are horizontal-branch stars. The horizontal branch is composed of red stars only. The age of the cluster, determined by comparison with the isochrones of Bell and Vanden Berg (1987) is consistent with an age between $12-14 \mathrm{Gyr}$. and a metallicity $[\mathrm{Fe} / \mathrm{H}]=-0.79$. The observed luminosity function shows a deficiency of stars from about $0.5 \mathrm{mag}$ below turnoff up to $g=21$ mag. A distance modulus of $(m-M)_{g 0}=15.35 \pm 0.1$ magnitude has been derived. An estimate of the cluster structural parameters gives $r_{c}=1.7 \mathrm{pc}$ and $c=1.46$. A mass estimate of $1.6 \times 10^{3} M_{\odot}$ and mase-to-light ratio 1.77 has been obtained, noing King's (1966) method. References

Bell, R., Vanden Berg, D. (1987) A\&AS, 63, 335

King, I. (1966) AJ, 71, 64

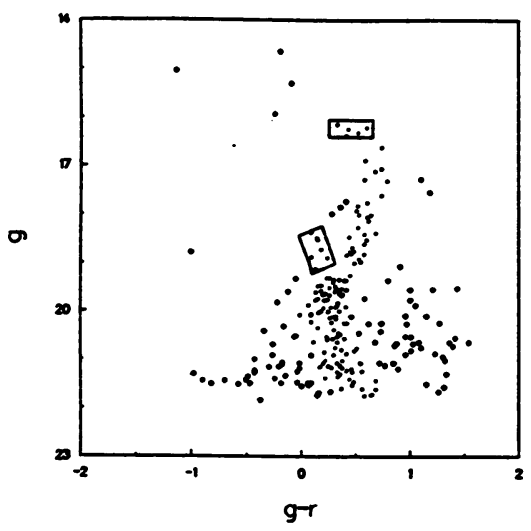

Figure 1. The observed g ve. g-r color-magnitude diagram of Pall 